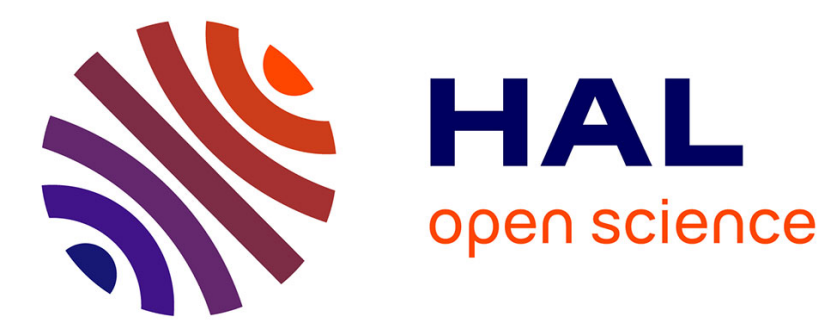

\title{
Une phase de sédimentation alluviale au Préboréal initial dans la vallées de l'Escaut (Nord de la France)
}

Laurent Deschodt

\section{To cite this version:}

Laurent Deschodt. Une phase de sédimentation alluviale au Préboréal initial dans la vallées de l'Escaut (Nord de la France). Quaternaire, 2002, 13 (2), pp.149 - 152. 10.3406/quate.2002.2188 . hal01489295

\section{HAL Id: hal-01489295 https://hal-inrap.archives-ouvertes.fr/hal-01489295}

Submitted on 3 Mar 2019

HAL is a multi-disciplinary open access archive for the deposit and dissemination of scientific research documents, whether they are published or not. The documents may come from teaching and research institutions in France or abroad, or from public or private research centers.
L'archive ouverte pluridisciplinaire HAL, est destinée au dépôt et à la diffusion de documents scientifiques de niveau recherche, publiés ou non, émanant des établissements d'enseignement et de recherche français ou étrangers, des laboratoires publics ou privés. 


\author{
UNE PHASE DE SÉDIMENTATION ALLUVIALE \\ AU PRÉBORÉAL INITIAL \\ DANS LA VALLÉE DE L'ESCAUT (NORD DE LA FRANCE)
}

\title{
RÉSUMÉ
}

A Fresnes-sur-Escaut (Nord), un transect partiel des alluvions en rive droite de l'Escaut se singularise, sous les dépôts limoneux, tourbeux et tuffacés holocènes, par un épais dépôt sableux lité à restes végétaux $(3 \mathrm{~m})$, dont la moitié supérieure est datée de la transition Tardiglaciaire-Holocène ou du tout début de l'Holocène ( $10040 \pm 50 \mathrm{BP})$. Il pourrait s'agir d'une accumulation sédimentaire rapide (favorisée par des facteurs locaux) en réponse à l'oscillation climatique majeure qui marque le début de l'Holocène.

Mots-clefs : Tardiglaciaire, Holocène, Prẻboréal, environnements fluviatiles, Escaut, Haine, Nord de la France.

\begin{abstract}
HIGHLIGHTING A ALLUVIAL SEDIMENTATION PHASE DURING EARLY PREBOREAL IN THE ESCAUT (SCHELDE) VALLEY (NORTHERN FRANCE)

At Fresnes-sur-Escaut, a partial cross-section of the Schelde's right bank alluviums is marked out, under the holocene silt, peat and calcarous tufa deposits, by a thick sandy bedded deposit with vegetal remains $(3 \mathrm{~m})$, of which the higher half is dated from the Lateglacial-Holocene transition or the early Holocene ( $10040 \pm 50 \mathrm{BP}$ ). It could be a quick sedimentary accumulation (favored by local factors) in response to the major climatic oscillation pointing out the begining of the Holocene.
\end{abstract}

Key-words : Lateglacial, Holocene, Preboreal, fluvial environnements, Escaut (Schelde) River, Haine River, Northern France.

\section{1 - INTRODUCTION}

L'Escaut entre à l'extrémité occidentale du bassin de Mons (Belgique) dans une vaste plaine alluviale humide, à environ $17 \mathrm{~m}$ d'altitude. Le fleuve y reçoit les eaux de la Haine. Leurs alluvions sont entourées de reliefs modérés développés dans un substrat crétacé (craie, marne) et paléogène (sable, argile). Très étendues, elles ont pourtant été peu étudiées, à l'exception notable de levés détaillés effectués sur des coupes visibles dans les berges érodées du lit mineur du fleuve, en amont de la confluence (Valenciennes, Saint-Saulve, Fresnes-sur-Escaut) et en aval (Bruilles-Saint-Amand) (Masson et Vallin, 1988). Les analyses palynologiques et datations ${ }^{14} \mathrm{C}$ associées (Ruchard et al., 1992) offrent une première syn- thèse de l'évolution du fond de vallée depuis le Boréal. Nos propres investigations ont été effectuées à la tarièregouge, en rive droite de l'Escaut (fig. 1).

\section{2 - STRATIGRAPHIE}

La stratigraphie des alluvions étudiées varie très peu le long du transect. Elle peut être synthétisée en trois grandes unités sédimentaires (fig. 2 et 3 ). De haut en bas :

\section{1 - DÉPÔTS FINS EN SURFACE}

Sous un horizon humifère peu épais (A), parfois labouré, un limon argileux ocre à taches rouilles (ARG) passe de manière diffuse à un limon argileux noir (AN).

* Institut National de Recherches Archéologiques Préventives, 518 rue Saint-Fuscien, F-80000 AMIENS, et HALMA EA 2457, atelier “ Paléoenvironnements et occupations humaines dans la vallée de la Deûle ”, Université Charles de Gaulle - Lille 3, F-59655 VILLENEUVE D’ASCQ. 


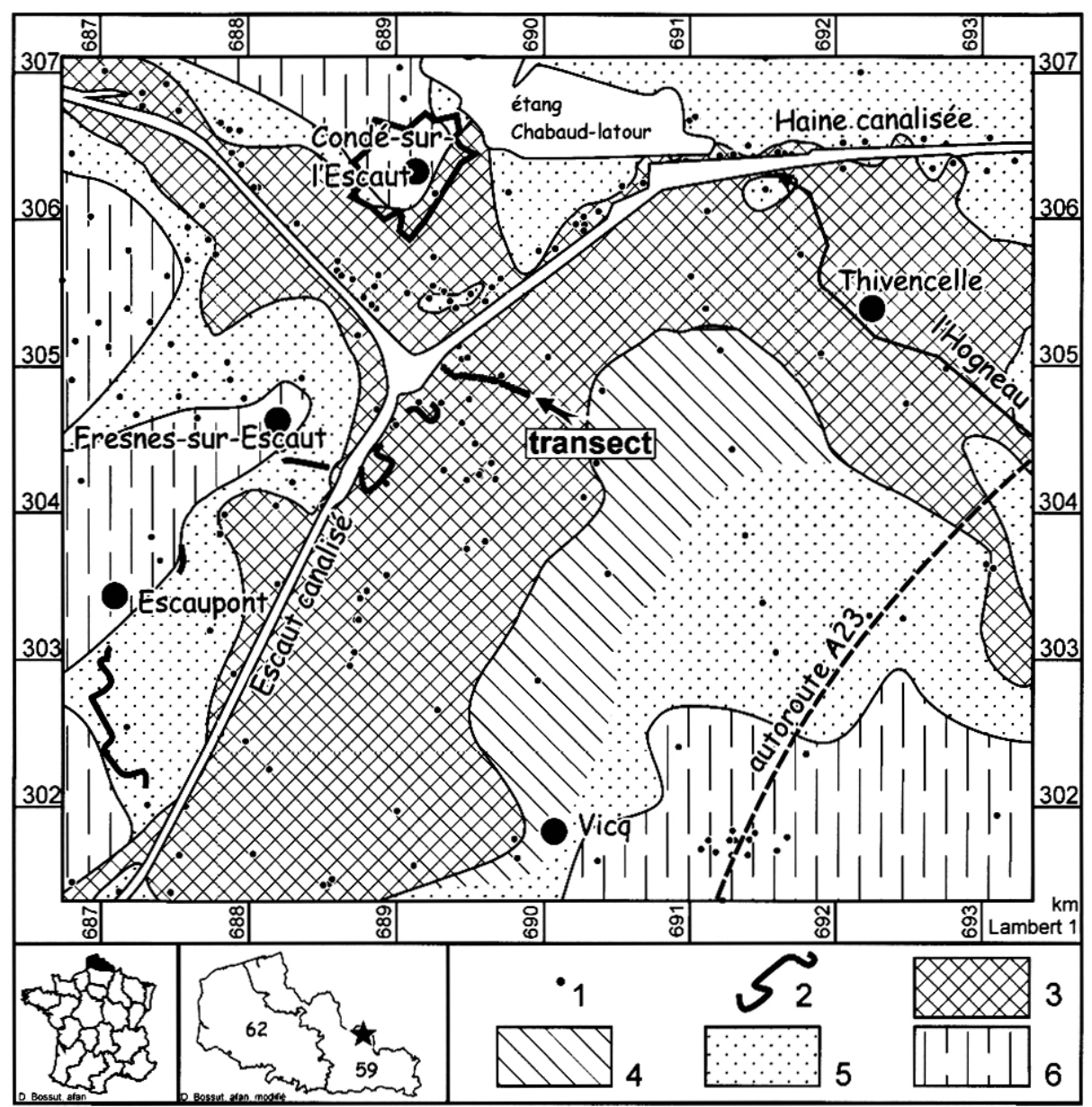

Fig. 1 : Localisation du transect (esquisse des formations quaternaires, d'après les sondages géotechniques).

Fig. 1 : Location of the cross-section (outlines of the quaternary formations, according to the geotechnic borings).

1 - sondage enregistré au BRGM - boring recorded by $B R G M$.

2 - méandre historique de l'Escaut visible sur la carte IGN 1/25000 - Scheld's historical meander visible on I/25000 IGN map.

3 - complexe de tourbe et de tuf calcaire holocène - holocene peat and calcareous tufa complex.

4 - description de " terre végétale " épaisse assimilable à un développement tourbeux - description of thick "vegetable ground " comparable to a peaty development.

5 - dépôt sableux de fond de vallée et jonction avec les versants - foodplain and floodplain/slope junction sandy deposit.

6 - formation limoneuse et limono-sableuse de versant - silt and silt-sand formation on slope.

L'épaisseur de l'ensemble, de l'ordre de $1 \mathrm{~m}$, augmente à proximité du lit mineur historique (environ $2 \mathrm{~m}$ ).

\section{2 - COMPLEXE DE TUFS ET DE TOURBES}

Sous les sédiments fins superficiels, du tuf calcaire, déposé en lits de gravier, de sable ou de limon, est associé à un sédiment tourbeux (TT) (épaisseur variable, de 0,7 à $2 \mathrm{~m}$ ). L'unité contient d'abondants mollusques aquatiques et de fréquents petits charbons de bois. Elle surmonte toujours une tourbe limoneuse litée $(\mathrm{Tb})$ datée de $7250 \pm 80$ BP (Beta 158173, sur bois) (épaisseur assez constante, de l'ordre de 0,1 à $0,25 \mathrm{~m}$ ).

\section{3 - DÉPÔT FLUVIATILE LIMONEUX ET SABLEUX}

La tourbe présente une limite nette (hiatus) avec un limon plastique noir (LPN), peu épais (environ 0,2 m), développé au-dessus d'un limon sableux gleyifié (LSG) ( 0,7 à $1,5 \mathrm{~m}$ d'épaisseur) avec de discrets litages sablonneux et horizons gris épisodiques. Une malacofaune est présente.
La limite inférieure de LSG est difficile à établir en sondage. L'unité passe à des lits fins et réguliers de sable et de sable limoneux (SL), gris-verdâtre à gris-brunâtre, épais (jusqu'à plus de trois mètres). Les lits les plus sombres sont limoneux et légèrement organiques, avec de petits débris végétaux (datation AMS sur bois, $10040 \pm$ 50 BP, Beta 158474, fig. 2 et 3). Des mollusques aquatiques sont présents et, épisodiquement, des restes de microfaune (dents de rongeur). Un cailloutis de silex, gélifractés et usés, parfois assez volumineux (plusieurs centimètres) est fréquent. En profondeur, un lit franchement tourbeux a été repéré (sondages 17 et 22, à environ $6 \mathrm{~m}$ sous la surface).

\section{4 - LES FORMATIONS SOUS-JACENTES}

Le sondage 22 (fig. 3) a permis d'observer le passage de SL à une série de lits plus limoneux, bleutés, sans autre modification que la texture et la couleur. Les sondages géotechniques (SOREG 1998) confirment la présence de nappes grossières (graveleuse et sablo-graveleuse) sur le bed-rock crayeux. L'incision maximum se 
situe sous la cote $5 \mathrm{~m} \mathrm{IGN} 69$ (environ $12 \mathrm{~m}$ d'alluvions quaternaires).

\section{3 - INTERPRÉTATION CHRONOLOGIQUE}

\section{1 - CORRÉLATION DE LA PARTIE SUPÉRIEURE DU TRANSECT AVEC LES DONNÉES LITHOLOGI- QUES, PALYNOLOGIQUES ET ${ }^{14} \mathrm{C}$ LOCALES}

La lithostratigraphie et la datation disponible à la base des sédiment tourbeux (unité $\mathrm{Tb}$ ) permettent une corrélation de la partie supérieure du transect avec les données locales (fig. 2) (Ruchard et al., 1992).

- Au Boréal, une sédimentation limoneuse ou limonosableuse progressivement organique est suivie de la formation d'une tourbe limoneuse $(8,1$ et $8,5 \mathrm{Ka} \mathrm{BP}$ à Saint-Saulve II) : corrélation avec l'unité LSG (ou de sa partie supérieure) et LPN.

Fig. 2 : Log schématique et palynozones probables, d'après les datations $14 \mathrm{C}$ et des corrélations stratigraphiques locales.

Fig. 2: Schematic log and likely palynozones, according to $14 \mathrm{C}$ datings and local stratigraphic correlations.

A - horizon superficiel - surface horizon.

ARG - limon argileux - clayey silt.

AN - limon argileux noir - black clayey silt.

TT - tufs et tourbes - tufas and peats.

$\mathrm{Tb}$ - tourbe - peat.

LPN - limon plastique noir - black plastic silt.

LSG - limon sableux gleyifié - gleish sandy silt.

SL - sable lité - bedded sand.

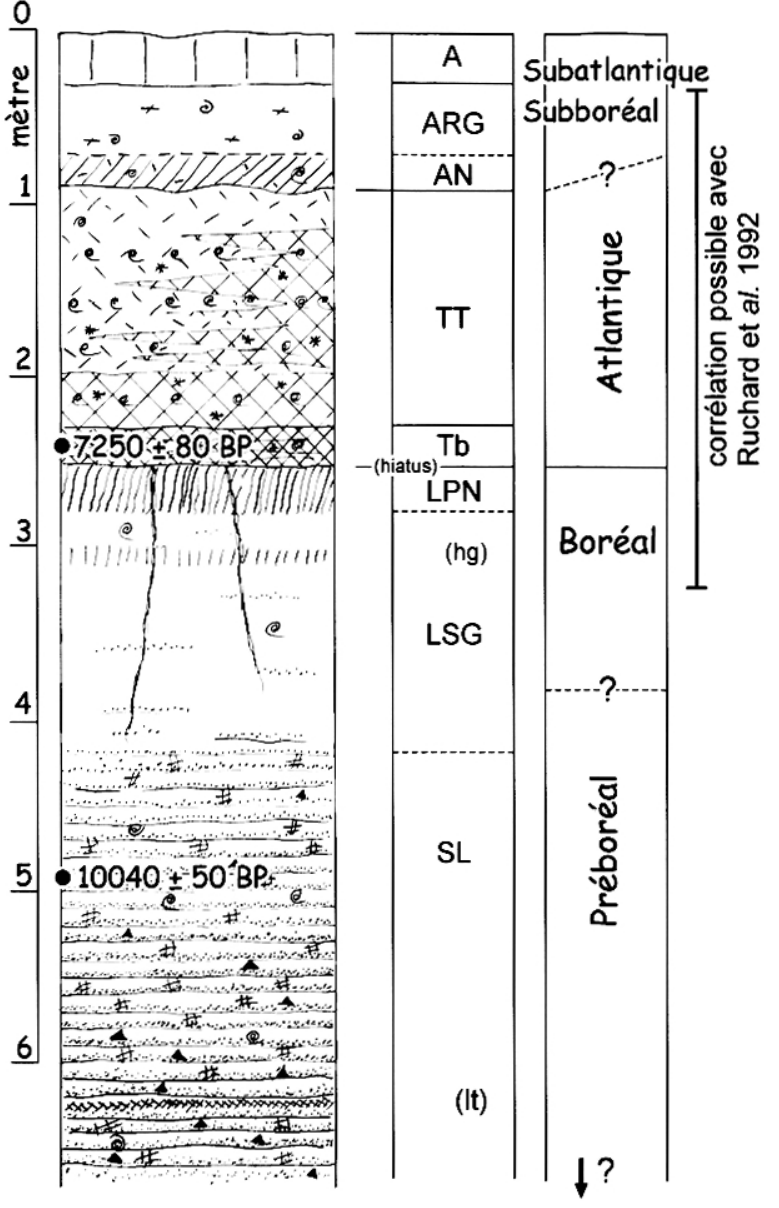

EST

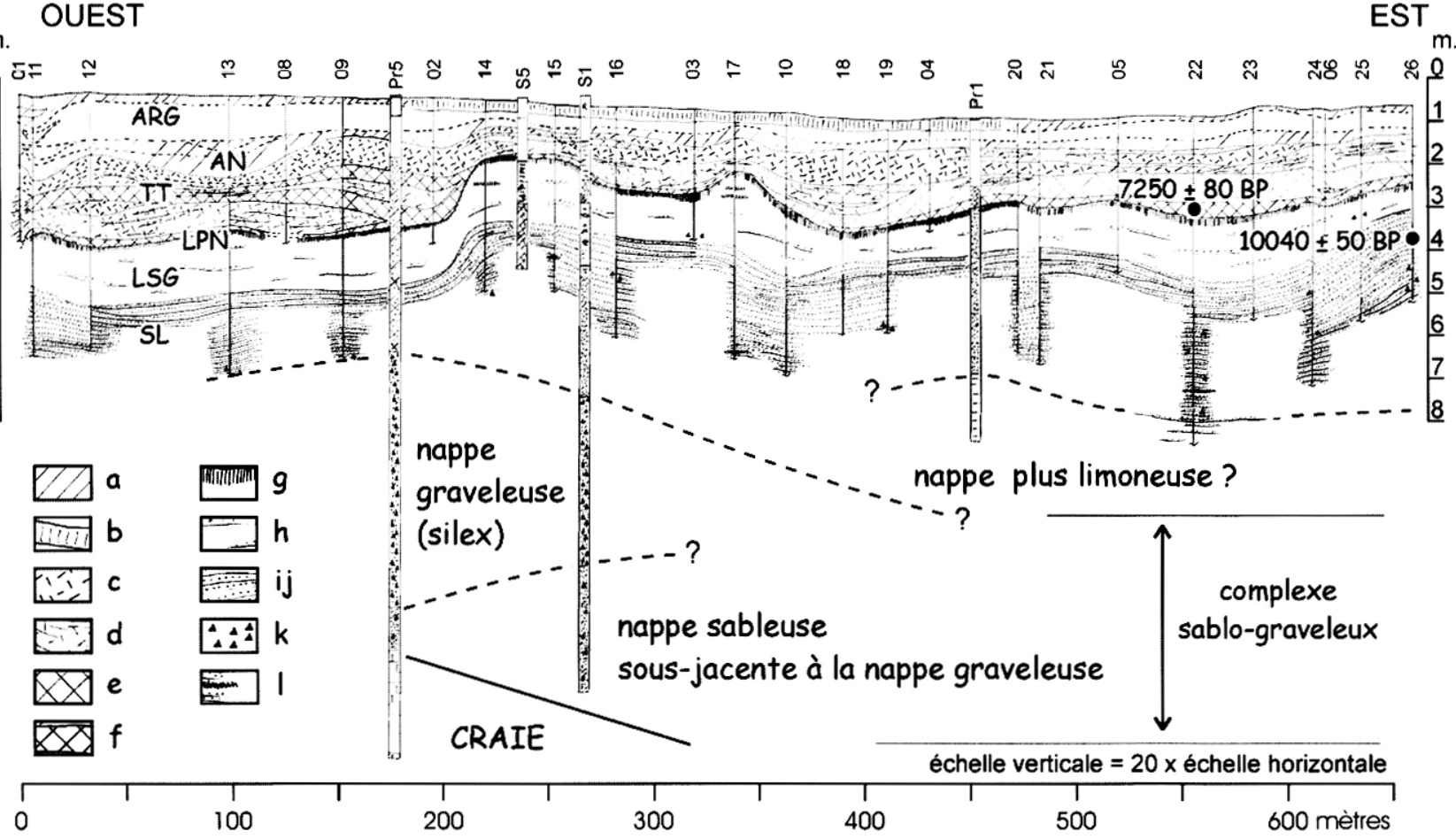

Fig. 3 : Transect est-ouest (avec projection des sondages géotechniques proches) et localisation des bois datés par radiocarbone.

Fig. 3 : East-west transect (with projection of close geotechnic borings) and location of radiocarbon dated woods.

a - horizon humifère - humic horizon.

$\mathrm{b}$ - labour (Ap) - ploughing horizon (Ap).

$\mathrm{c}$ - tuf grossier - coarse tufa.

$\mathrm{d}$ - tuf fin (sable et limon) lité - bedded fine (sand and silt) tufa.

e - lits de limon tourbeux, plus ou moins tuffacé - peaty silt beds with more or less tufa.

$\mathrm{f}$ - tourbe franche et limon tourbeux - peat and peaty silt.

$\mathrm{g}$ - horizon noir argileux - clayey black horizon.

$\mathrm{h}$ - limon gleyifié à lits sableux sporadiques - gleish silt with sporadic sandy beds.

ij - lits sableux - sandy beds.

$\mathrm{k}$ - cailloutis de silex - flint gravels

I - passée tourbeuse - peaty bed. 
- A l'Atlantique, la sédimentation organique se poursuit, sous forme de tourbe limoneuse $(7,1 \mathrm{Ka}$ BP à SaintSaulve II) : corrélation avec l'unité Tb (7 $250 \pm 80$ BP).

Par la suite, les alluvions sont constituées de limon humifère coquillier (7,6 Ka BP à Fresnes II), de tourbe (5,9 et 5,5 Ka BP à Valenciennes), puis de travertin : corrélation avec l'unité de tourbes et tufs TT.

- Au cours du Subboréal-Subatlantique, la plaine alluviale semi-boisée fait place à une prairie humide. La sédimentation devient non humifère (limon de débordement) (datation du sédiment humifère le plus tardif, sur un limon coquillier, à Fresnes I : 3,3 Ka BP) : corrélation avec ARG.

\section{2 - DATATION DE L'UNITÉ SL}

La datation sur bois dans l'unité SL (10 $040 \pm 50 \mathrm{BP}$, soit en années calendaires : $11610 \mathrm{BP} \mathrm{Cal}$, intervalle $2 \sigma 11940$ - $11280 \mathrm{BP}$ Cal) correspond à l'oscillation climatique rapide qui marque le début de l'Holocène dans les glaces des sondages arctiques (réchauffement sensible aux hautes latitudes vers $11645 \mathrm{BP}$ Cal, confirmé et stabilisé vers 11612 BP Cal, Taylor et al., 1997). Si nous accordons confiance à cette seule datation sur bois, l'unité " SL" se trouve donc à la transition Tardiglaciaire-Holocène ou au tout début de l'Holocène.

\section{CONCLUSION}

A Fresnes-sur-Escaut, un élément remarquable de la stratigraphie des alluvions est la présence, à la base de la séquence holocène, de lits sableux réguliers et étendus, à débris végétaux, datés de la transition Dryas récentPréboréal ou du tout début du Préboréal. L'épais dépôt se distingue de la plupart des enregistrements des fonds de vallée où au basculement climatique Weichsélien-Holocène répond une phase d'incision des chenaux suivit d'un comblement, tourbeux, plus tardif de quelques décennies :

- 9,0 Ka BP à Maisière-Canal, Haisne (Haesaerts, 1984) (environ $25 \mathrm{~km}$ en amont de la zone étudiée),

- 9,9 Ka BP dans le Bas-Escaut (Verbruggen et Kiden, 1989) (une centaine de $\mathrm{km}$ en aval de la zone étudiée),

- 9,9 à 9,8 Ka BP dans la Somme (Antoine, 1997a, ; Antoine et al., 2000 ; Ducrocq, 2001),

- 9,8 à 9,7 Ka BP, dans les vallées de l'est du Bassin Parisien (Pastre et al., 1997 ; Pastre et al., 2000).

Toutefois, dans le bassin de la Somme, les premières tourbes holocènes de fond de chenal sont précédées d'un limon fluviatile faiblement organique (communication $P$. Antoine) (Etouvie, Antoine, 1997a ; Conty dans la vallée de la Selle, Antoine, 1997b). Ce premier dépôt peut être parfois accompagné d'une charge en graviers (Conty). La précocité de la sédimentation souligne la rapidité de l'incision, et du colmatage, au Préboréal initial.
A Fresnes-sur-Escaut, l'unité “ SL " bénéficie d'une datation absolue. Le développement important (tant en épaisseur qu'en étendue) de l'unité a pu être favorisé par des facteurs locaux : morphologie fluviale dans une vaste zone de confluence, abondance de la charge sableuse... Son faciès évoque une aggradation régulière et rapide que d'autres datations pourraient confirmer. Dans ce cas cllc constitucrait un témoin remarquable de l'évolution environnementale du Préboréal initial.

\section{REMERCIEMENTS}

Je remercie P. Antoine et J. Sommé pour leurs conseils, P. Mathys pour son aide sur le terrain et $J$. Denby pour la correction du résumé anglais.

\section{BIBLIOGRAPHIE}

ANTOINE, P. 1997a - Modifications des systèmes fluviatiles à la transition Pléniglaciaire-Tardiglaciaire et à l'Holocène : L'exemple du bassin de la Somme (Nord de la France), Géographie physique et Quaternaire, 51 (1), 93-106.

ANTOINE, P., 1997b - Evolution Tardiglaciaire et début Holocène des vallées de la France septentrionale : nouveaux résultats, Compte rendu de l'académie des Sciences de Paris, Sciences de la Terre et des Planètes, 325, 35-42.

ANTOINE, P., FAGNART, J.-P., LIMONDIN-LOZOUET, N. et MUNAUT, A.-V., 2000 - Le Tardiglaciaire du Bassin de la SommE : éléments de synthèse et nouvelles données, Quaternaire, $11(2), 85-98$

DUCROCQ, T., 2001 - Le Mésolithique du bassin de la Somme, Publications du Centre d'Etudes et de Recherches Préhistoriques, 7,253 p.

HAESAERTS, P., 1984 - Les formations fluviatiles Pléistocènes du bassin de la Haine (Belgique). Signification dynamique et climatique des formations et terrasses fluviatiles quaternaires. Colloque de l'AFEQ, Paris, 29 janvier 1983, Bulletin de l'Association Française pour l'Etude du Quaternaire, 1.2.3, 19-26.

MASSON, B. et VALLIN, L., 1988 - Recherches sur la plaine alluviale de l'Escaut (1984-1986). Paléoenvironnement et Archéologie, Cahiers de la Préhistoire du Nord, 3, 23-33.

PASTRE, J.-F., FONTUGNE, M., KUZUCUOGLU, C., LEROYER, C., LIMONDIN-LOZOUET, N., TALON, M. et TISNÉRAT, N., 1997 - L'Evolution tardi- et postglaciaire des lits fluviaux au nord-est de Paris (France). Relations avec les données paléoenvironnementales et l'impact anthropique sur les versants, Géomorphologie : relief, processus, environnement, 4, 291-312.

PASTRE, J.-F., LEROYER, C., LIMONDIN-LOZOUET, N., CHAUSSE, C., FONTUGNE, M., GEBHARDT, A., HATTE, C. et KRIER, V., 2000 - Le Tardiglaciaire des fonds de vallée du bassin Parisien (France), Quaternaire, 11, (2), 107-122.

RUCHARD, N., LENAERTS, S. et MUNAUT, A.-V., 1992 - Etude palynologique de sédiments holocènes dans la plaine alluviale du Haut-Escaut (Nord, France), Cahiers de la Préhistoire du Nord, 10, 118-130.

SOREG 1998 - dossier R-98-0406, juin 1998 (sondages PONTIGNAC).

TAYLOR, K.C., MAYEWSKI, P.A., ALLEY, R.B., BROOK, E.J., GOW, A.J., GROTTES, P.M., MEESE, D.A., SALTZMAN, E.S., SEVERINGHAUS, J.P., TWICKLER, M.S., WHITE, J.W.C., WHITHLOW, S. et ZIELINSKI, G.A., 1997 - The HoloceneYounger Dryas transition recorded at Summit, Greenland, Science, 278, 825-827.

VERBRUGGEN, C. et KIDEN, P., 1989 - L'Evolution postglaciaire du Bas et Moyen Escaut, Cahiers de la Préhistoire du Nord, 6, 5-14. 